\title{
PREDICTING THE STRENGTH OF LEONID OUTBURSTS
}

\author{
ESKO J. LYYTINEN \\ Kehäkukantie 3 B, 00720 Helsinki, Finland \\ E-mail:esko.lyytinen@minedu.fi \\ and \\ TOM VAN FLANDERN \\ Meta Research, 6327 Western Ave, NW, Washington, DC 20015, USA \\ E-mail: tvf@mindspring.com
}

(Received 27 June 2000; Accepted 18 August 2000)

\begin{abstract}
A simple model is described that predicts the time of occurrences and peak activity of Leonid shower outbursts. It is assumed that the ejection speeds of escaping particles at each return of the parent comet near perihelion are very small, but solar radiation pressure acting differently on different particles causing a spread of particles into different period orbits. Earlier papers predicted the position of the resulting dust trails. This paper sets forth to better predict the strength of the expected outbursts by considering the role of non-isotropic effects in the interaction with the solar radiation on the dispersion of particles away from the dust trail center. This paper determines the approximate magnitude of the relevant effects. Predictions for the next few years are presented that include such considerations, for reasonable assumptions of particle properties. For example, earlier predictions for the 1999 storm of ZHR $=6,000-7,000$ are now reduced by a factor of two, which is in better agreement with the observed ZHR 4,000. The success of the technique, when applied to historic meteor storms and outbursts without need of additional free parameters, lends confidence to the soundness of the underlying model and to its application for future predictions. We predict that the best encounters of this return of the parent-comet will occur in the years 2001 and 2002.
\end{abstract}

Keywords: Comet, comet ejection, Leonids, meteor, meteor shower, meteoroid, model predictions, orbital dynamics, radiation pressure

\section{Introduction}

This work was initiated in early 1999 after the 1998 fireball outbursts arrived unexpectedly and far "off-schedule" (Lyytinen, 1999). Its premise, low ejection velocities, is based on the satellite model of comets, which was developed earlier (Van Flandern, 1981; 1999). In that model, a comet is supposed to have a nucleus with an orbiting debris cloud system. The mass of the debris cloud can be comparable 
to, or in excess of, the mass of the primary nucleus. It is postulated that at each perihelion there is gravitational escape of particles through the L1 and L2 Lagrangian points. Smaller particles, i.e., most meteor size particles, are actually stable only in inner orbits around cometary nuclei, and are driven out from orbits around the cometary nucleus mainly by radiation pressure. Slightly different, but regarding the formation and course of trails, essentially similar assumptions have been made in earlier work by Kondrat'eva and Reznikov (1985) and Kondrat'eva et al. (1997) and recently in the work by McNaught and Asher (1999). The first to suggest the importance of radiation pressure was Kresak (1976). The first to realize the formation of trains due to different orbital periods was Pavel (1955).

If the ejection speeds are very small with little dispersion and the orbital dispersion is caused mainly by solar radiation pressure, then the radiation pressure acts as if solar gravitation were decreased, thereby increasing the semi major axis, thus also the period of revolution, according to the particle size. That increase is proportional to the radiation pressure/mass ratio. As a result of differences in orbital period, the particles form trails after one revolution.

Transverse spread is caused by the pressure of non-symmetric absorption and re-radiation of solar radiation. It is the spread along the comet orbit and the transverse spread that are responsible for the observed intensity of the shower. This is usually expressed in terms of Zenith Hourly Rate (ZHR). Our predicted peak rate prior to the1999 storm was ZHR $=6,500 \pm 500 \mathrm{hr}^{-1}$ (Lyytinen, 1999). After the observations of the storm suggested a peak activity of ZHR $\sim 4,000 \mathrm{hr}^{-1}$ (Arlt et al., 1999; Jenniskens et al., 2000), we re-addressed earlier concerns on such non-isotropic effects of the interaction of the meteoroids with sunlight. In this paper, we will introduce a better treatment of the radiation pressure effects to arrive at more reliable estimates of the peak activity of future meteor storms. Some other refinements of the model are also introduced.

\section{Method}

The backward integration of the parent comet was conducted as in Yeomans et al. (1996), adjusted to agree with observed returns since 1366. From each perihelion a number of particles (typically about 140) with different radiation pressure for each were integrated forward. The radiation pressure introduces an effective decrease of the gravitational pull for each particle. We recorded the time, radius vector and longitude at the descending node crossings of the 1950.0 ecliptic plane of each particle. Figure 1 gives a graphic presentation of the trails around the present return of the parent comet. The data was manually checked for encounters with the Earth.

Corrections for the true (mean) Earth orbital plane of the date were made for longitude and for the radius vector. Our earlier work did not include the radius correction (Lyytinen, 1999). The heliocentric distance of ecliptic crossing $r_{D}$, is affected in the fifth decimal. In 
comparison to our earlier model, the perihelion distance $\mathrm{q}$ of the parent comet needs a reduction of $0.000077 \mathrm{AU}$ according to the most recent orbital elements by Nakano (1998) This brings the adopted orbit close to the value used in McNaught and Asher (1999a). So each $r_{D}$ value in the earlier results was reduced by this amount.

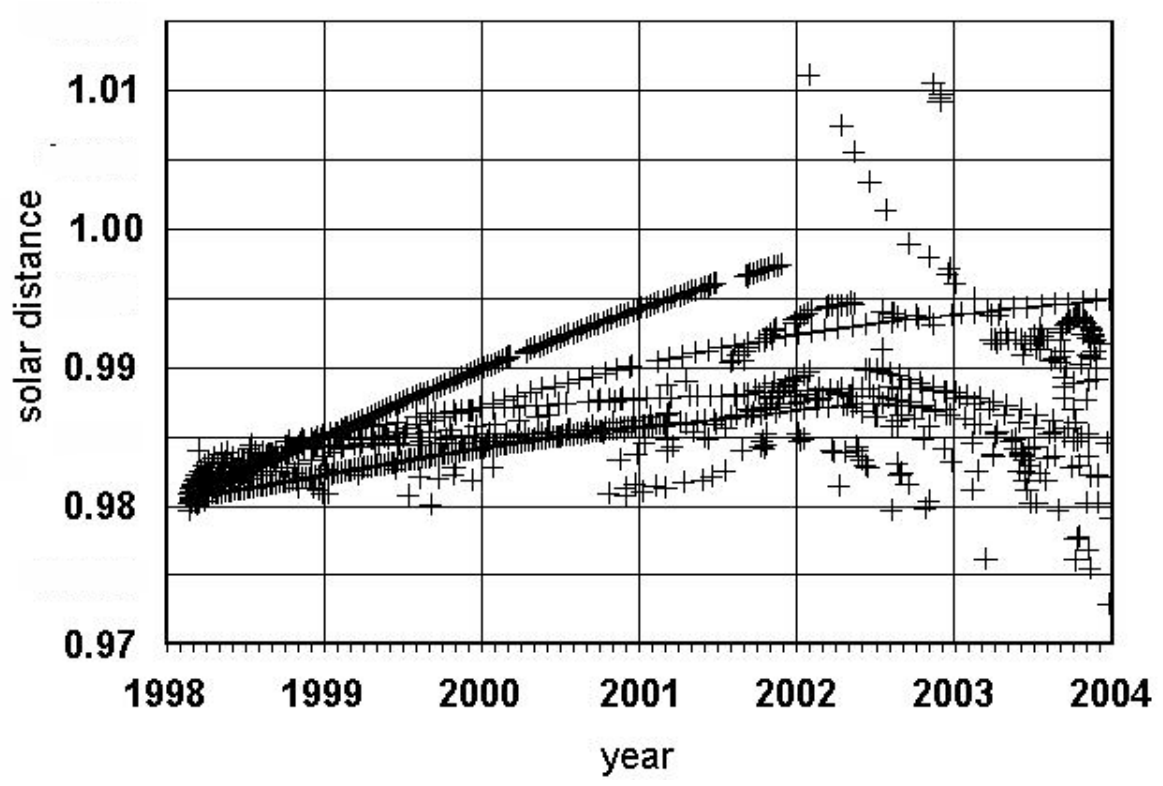

Figure 1. Distance from the Sun of modeled trail particles at the descending node. The vertical lines are at the start of January. The Earth-Sun distance is at about 0.988 to 0.989 AU. A concentration of points near this value in mid-November (as in the year 2001) indicates a likely meteor storm.

\section{Treatment of radiation pressure}

What is usually called simply radiation pressure is here called "regular radial pressure". It is a repulsive force, which is inversely proportional to solar distance, effectively lowering the gravitational force. Other radiation pressure effects can be non-radial and can have a different dependence on solar distance. Notably, variations in radiation force as a result of the particle's surface to mass ratio, rate of rotation, etc., will lead to dispersion of particles, effectively widening the trail perpendicular to the Earth's orbit and in the Earth's orbital plane. For the purpose of calculating the shower's activity, one can group the various effects according to their influence on the dispersion of the stream. The general term radiation force is used to cover all the effects that describe the particle's response to solar radiation. 


\subsection{THE REGULAR RADIAL PRESSURE}

The radiation force on a particle consists of three parts: due to absorption of solar radiation energy, due to re-radiation of the thermal energy, and due to reflection (scattering). The radiation pressure force on a totally absorbing particle of diameter $\mathrm{d}$, neglecting the other two effects, at a distance $r$ from the Sun is:

$$
\mathrm{F}_{\mathrm{r}}=\pi(\mathrm{d} / 2)^{2} \mathrm{~S}_{\mathrm{o}} \mathrm{c}^{-1} \mathrm{r}^{-2}
$$

The constant $\mathrm{S}_{0}$ is the solar radiation constant at Earth's distance and c is the light speed.

In the ideal case where there is no absorption and all the energy would be reflected directly backwards (to the Sun) like a flat mirror put normal to the solar radius, the total effect would be twice that given by (1). Similarly, in the ideal case that all the energy would be re-radiated as thermal radiation directly backwards toward the Sun, the force by reradiation would be the same as that of scattering. Hence, if the particle is non-rotating, spherical, non-conducting and homogenous on its surface, we can assume that the total force due to absorption, reradiation, and scattering is directed away from the Sun and obeys an inverse square law. Particles affected by solar radiation pressure in this manner are effectively in a weaker gravitational field. The motion of the particle without further perturbations is a Keplerian ellipse. Because the particles are assumed to escape into their own orbits near the perihelion of the comet's orbit, radiation pressure has the effect of creating a slightly wider elliptic orbit, with a slightly bigger semi-major axis, and longer orbital period.

The ratio of radiation pressure to gravity $(\beta)$ for similar particles is inversely proportional to the particle diameter. It is mostly affected by the surface-to-mass ratio of the particles. So different-sized particles get into elliptic orbits with different orbital periods, thereby forming elongated trails without much tendency to spread perpendicular to the orbit.

Non-spherical particles can have different average surface-to-mass ratio, so actually it is expected that to some degree different mass particles will exist even with similar orbital periods. This also applies to particles with different densities.

The net effect of the radial component of the radiation pressure is to distribute the particles along the comet orbit and delay the population of particles relative to the comet after even a single orbit. Subsequent orbits cause this dispersion to increase by about the same amount each return. In the case of Leonid meteoroids, a radiation pressure of one thousandth of the gravity of the Sun will give a period of revolution of 380 days longer than that of the parent comet (which has an orbital period of about 33.3 years). The force of radiation pressure as 
described by (1) on a one-millimeter-diameter sphere with density $1 \mathrm{~g}$ $\mathrm{cm}^{-3}$ is about this big.

Key to the predicting encounter times for Leonid outbursts is the point where the so formed dust trail crosses the ecliptic plane.

\subsection{PERTURBATIONS}

The inverse-square and radial force law of Equation 1 is the starting approximation. All departures from this law are perturbations, that are discussed in the next sections. Such perturbations affect the shape of the orbit and the ecliptic plane crossing distances away from the Sun. Because the Leonid meteoroids are observed near the perihelion of their orbits, perturbations on the total angular moment arising far away from perihelion are of this type. The net effect is a dispersion of particles giving rise to the shape of the Zenith Hourly Rate (ZHR) activity curve of the meteor storm.

The purpose of this section is to show that there exist mechanisms that can quantitatively explain the scatter of particles, to the extent that it is sensible to construct a ZHR model.

\subsubsection{The "A2 effect"}

A non-graviational induced period change, over the regular radiation pressure and with little effect otherwise, is called here the "A2" effect, because of an analogy with non gravitational effects in comet orbital dynamics. The "A2" effect is in particular caused by perturbations on the speed of the particles due to a perturbing component along the plane and normal to solar radius that arises near perihelion. Such perturbation mainly affects the orbital period but hardly at all the ecliptic plane crossing radius. The Poynting-Robertson effect is an example of such a perturbation, even though very small. In the Poynting-Robertson effect, radiation is emitted with higher momentum towards the direction of motion, effectively slowing down the grain. The "A2 effect" by the other mechanisms described in this paper can be to either direction, at least over a short time span.

These perturbations do not directly affect the meteor outburst predictions because the particles that would encounter the Earth are replaced by others with similar ecliptic plane crossing radii and nodes. Only the orbital period determines whether particles reach the ecliptic when the Earth is nearby. The true effect of such perturbations is to change somewhat the encounter conditions and cause slightly altered differential planetary perturbations over its orbit. The magnitude of such perturbations is comparable with the radial effect for old trails and cannot be separated in a straight-forward manner. The spread of particles by this effect can be modeled by computer simulations that include planetary perturbations. The A2 effect mainly affects encounters with old trails. 


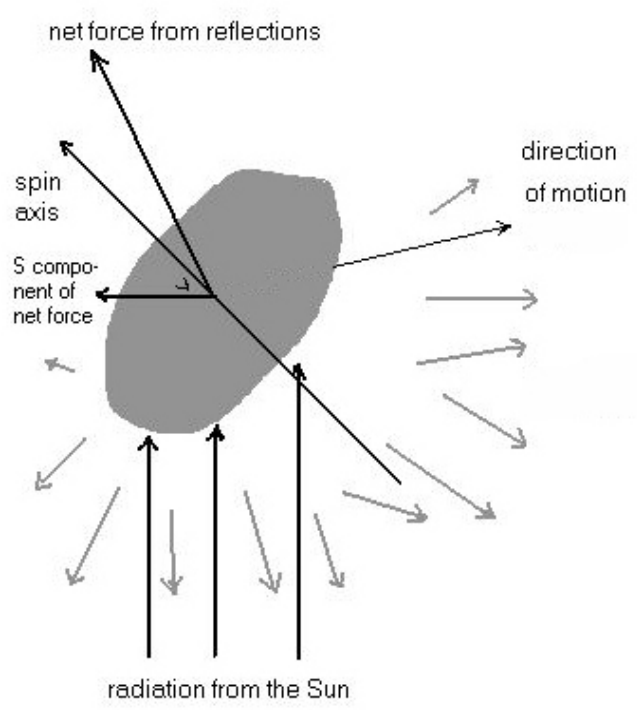

Figure 2. Non-isotropic scattering may give force components that change the orbit and, among others, affect the orbital period, i.e. produce an A2 effect.

\subsubsection{Non-isotropic scattering of radiation}

Consider the perturbing forces from radiation scattered nonisotropically from a dust grain in three directions: $\mathrm{R}$, along the radius vector away from the Sun; W, normal to the orbital plane; and S, in the orbital plane towards the direction of motion and normal to the previous directions (Figure 2a). For both the components R and S, there appears a $\sin (2 *(v+$ constant $))$ type of dependency on the direction of the force (where $v$ is the true anomaly; with a different constant for each particle and force component). This effect mostly concerns flattened particles, but can arise even for spherical particles with non homogenous surfaces. An imaginary extreme example would be a two-sided mirror such as a solar sail. This mechanism perturbs the period, but during one orbit the net is zero. This type of perturbation does not cause a net change of orbital period if there is no further dependency of solar distance $r$ than the normal $1 / \mathrm{r}^{2}$. The conclusion of no orbital period change with this type of perturbation was derived from a simple program integrating the effect of the proposed mechanism during one orbit, running the program for various different spin axis directions of the particle. A test particle with the regular radiation pressure of one thousandth of solar gravity $(\beta=0.001)$ was used. It may be that the true dependency of these components is more complex than in the studied example and may cause some A2 effect as well. 
This effect, even in the pure form, does cause a dispersion. This arises mostly from perturbations near aphelion, even though $1 / \mathrm{r}^{2}$ reaches its minimum there. Assuming that the component $\mathrm{S}$ is one hundredth of the total radiation pressure at aphelion, and that the test particle further obeys the "double sine law" $\sin (2 * v+$ constant) (or in this case $\cos \left(2^{*} v\right)$ ), we find that our test particle above gives a shift in $\mathrm{q}$ of $\pm 0.00016 \mathrm{AU}$ during one revolution. This applies only to a high albedo particle. If the particle albedo is small, this effect may be too small to explain much of the observed particle dispersion in meteor storms.

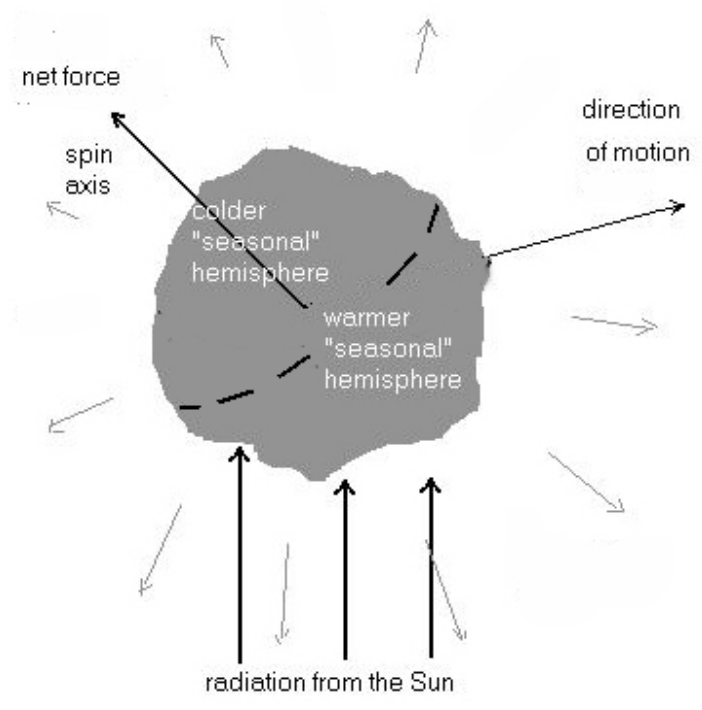

Figure 3. The mechanism for seasonal Yarkovsky is illustrated here. This effect can perturb the orbital motion of a particle much like the effect illustrated in Figure 2.

\subsubsection{Non regular thermal re-radiation: the seasonal Yarkovsky effect}

Previous perturbations do not consider rotation of the particle and a delay of re-emission, causing the (diurnal) Yarkovsky effect. This effect arises from the fact that the there is a lag in the temperature of the surface during one revolution causing the temperature in the "afternoon" to be larger than at a similar phase during the "morning". Because the rotation of a small body is quite fast, the diurnal Yarkovsky force is rather small (Olsson-Steel, 1987). The effect depends on thermal lag and unknown rotation properties.

Instead, we will consider what is now called the "seasonal Yarkovsky", which was recognized by Rubincam (1995, 1998) and Farinella et al. (1998) (e.g. see Rubincam, 1995; Vokrouhlicky and Broz, 1999). Their treatment is for asteroid sized objects, down to 0.1 $\mathrm{m}$. The seasonal Yarkovsky effect arises from the tilt of the spin axis, 
which puts one hemisphere towards the Sun and the other away from the Sun. The thermal re-radiation from the "summer" hemisphere causes an effective force in the direction of the "winter" hemisphere, in a direction different from the radial direction towards the Sun. The effect depends on conveying thermal energy effectively to the night side within the diurnal period, by rotation. The effect does not depend on rotation properties (within a reasonable range of spin rates), but on distributions of the spin axis tilt. The strength of the seasonal Yarkovsky depends on a particle's thermal conductivity, as well as its size. For small grains, the seasonal effect is several orders of magnitude larger than the diurnal variant. The effect for asteroids is a modest semi major axis reduction (Rubincam, 1995; Vokrouchlicky and Broz, 1999).

We derived an approximate formula for this force in the case of spherical particles with a distribution of spin angles. We assume that the Sun's radiation is totally absorbed and calculate the difference in reemission from the day and night time sides. The radiation from the shadowed side is equal to the thermal energy going by conduction through the particle. The thermal conduction is proportional to the difference in temperature of the two hemispheres. We find for a particle whose spin axis is pointed towards the Sun:

$$
\mathrm{F}=\mathrm{J} \mathrm{S}_{\mathrm{o}} \mathrm{d}^{2}\left(\mathrm{c} \mathrm{r}^{2}\right)^{-1}\left(1+\mathrm{L}(\mathrm{K} / \mathrm{d}) \mathrm{r}^{3 / 2}\right)^{-1}
$$

where $\mathbf{J}$ is an (adjusting) factor, whose effect will get introduced in the numerical coefficient in equation (4), $\mathrm{K}$ is the thermal conductivity and:

$$
\mathrm{L}=2^{-1 / 4} \mathrm{~S}_{\mathrm{o}}^{-3 / 4} \sigma^{-1 / 4}
$$

where $\sigma$ the Stefan Bolzmann coefficient. Note that as thermal conductivity $\mathrm{K}$ approaches zero, this will give the distance dependency $1 / r^{2}$. Hence, the force from thermal re-radiation given by the seasonal Yarkovsky effect, in the case of a black body and axis directed to the Sun, is proportional to the gravity at the distance in question. Equation 2 can be re-written as:

$$
\mathrm{F}_{\mathrm{SY}}=0.18 \beta\left(1+\mathrm{L}(\mathrm{K} / \mathrm{d}) \mathrm{r}^{3 / 2}\right)^{-1}
$$

If the body is not black, the resulting forces must be multiplied by the absorption coefficient; i.e., with (1-albedo). The force components vary with $\sin (2 \mathrm{x}$ angle).

For the purpose of checking the accuracy of the approximate solution of non linear equations, we put equation (4) in the general form:

$$
\mathrm{F}_{\mathrm{SY}}=0.18 \beta(1+\mathrm{H})^{-1}
$$

where we treat $\mathrm{H}=\mathrm{L}(\mathrm{K} / \mathrm{d}) \mathrm{r}^{3 / 2}$ as one variable. The maximum proportional error is only about 4 per cent. The $\mathrm{H}$ range with this size error is around $0.05-0.4$, while it is smaller outside this range. This 
size error arises from the approximations in the solution of nonlinear equations. Approximations in the spherical shape geometry will introduce further errors, but the particles are not expected to be spherical in reality.

The ecliptic crossing of the Leonids is close to the perihelion of the orbit. So it was numerically computed how the Equation 4 force affects q. We assumed that the spin axis is situated in the orbital plane, keeping its orientation during one orbit and varying the direction in the plane with different runs to get some maximal values for the variability of $q$ by this effect. Within the same computer runs, the change in semi major axis was computed. This gives the possible change of the period of revolution in one orbit; i.e., the A2 effect.

With near-zero thermal coefficient $K$, the maximum change of $q$ during one revolution is about $0.003 \mathrm{au}$. The $\mathrm{A} 2$ effect is zero. When $\mathrm{K}$ increases, the change of q decreases quite rapidly while the A2 effect increases before reaching a maximum. At its peak, the orbital period change is about two weeks over one orbital revolution. Unfortunately, this is in the parameter range for $\mathrm{H}(0.05-0.4)$ where the assumptions that go into Equation 4 may break down. Olsson-Steel (1987) gives the value of $\mathrm{K}$ for terrestrial silicates as $\mathrm{K}=3.5 \mathrm{~W} \mathrm{~m}^{-1} \mathrm{~K}^{-1}$. The value of $\mathrm{K}$ for returned lunar samples is only about one percent of this. This should be further reduced by about one and a half orders of magnitude to get into the region of the biggest $\mathrm{A} 2$ effect (for a one $\mathrm{mm}$ particle). With the values of the lunar samples, an A2 effect a bit less than a day is reached. With this value, the change of $q$ is only about 0.00001 au. A change in $\mathrm{q}$ essentially translates directly to a change in the radial ecliptic plane crossing distance of the particle, $\mathrm{r}_{\mathrm{D}}$. This is too little to explain the direct spread of $r_{D}$ inferred from observations. Hence, the seasonal Yarkovsky effect will either change $q$ (and $r_{D}$ ), or create a larger dispersion along the orbit.

We have not studied the seasonal Yarkovsky effect normal to the plane, but the perturbing normal component has the dependency (at least approximate) of sin(angle-along-plane). This angle is from the node of the "particle equator" in the orbital plane. So this clearly has an effect on the plane and causes some scatter normal to the orbital plane.

In the case of a very flat particle, the mean surface to mass ratio is now bigger than with spherical particles having a given value of $\beta$ resulting in a bigger mass particle than a spherical particle with the same $\beta$ value. Further, for a sheet-like particle, the range of total radiation pressure force varies from its maximum value to practically zero. If the Sun shines normal to the particle spin axis, then the temperature of the particle falls. This actually gives a further reduction of the re-radiation force in the direction of the spin axis.

\subsubsection{Effects due to precession of the particle spin axis}

Olsson-Steel (1987) considered the effects due to precession of the particle spin axis for a particle with $1 \mathrm{~mm}$ radius, whereas we have it at 
$0.5 \mathrm{~mm}$. So it is quite close to this particle size. For an assumed spin rate, Olsson-Steel calculated the angular change of axis by precession as approximately $10^{-6} \mathrm{rad} \mathrm{s}^{-1}$ (at $1 \mathrm{AU}$ ). Although Olsson-Steel noticed that this would imply that the spin axis revolves around the Sunmeteoroid line several times in each orbit, we find a lower precession rate. Olsson-Steel takes as the force for torque the whole radiation pressure, which is clearly too coarse an approximation. Actually, for a spherical particle, the torque is zero both for absorption and thermal reradiation. This applies also for flattened elliptic particles, if we neglect diurnal effects and the Poynting-Robertson type effects are also neglected in all cases. For scattering, it is also zero for a sphere, but non-zero for elliptic flattened particles. With flattened particles, it may be reasonable to expect the precession rate to be about two orders of magnitude smaller than the value above. This would imply that the spin axis can remain more or less fixed in space for several orbital revolutions.

For the prediction of meteor storms, the assumption of fixed orientation is reasonable. However, at the extreme $\Delta$ a range (large initial difference in orbital period) as in the outburst of 1969, this is no longer true, but the model and predictions have not been altered for this. As a consequence, the predicted 2006 and maybe 2007 outbursts will have perhaps twice the ZHR given in Table I below.

\section{The meteor stream model}

From the previous considerations, we have constructed a stream model that is partly empirical by aiming to comply with past Leonid shower observations, but derives some properties from the assumed radiation pressure spread in section 3. For the most part, the form of the equations have a theoretical basis.

The spread into different orbital periods by the assumed regular radiation pressure is already embedded within the trail computations. These computations will also give the spread along the orbit that is expressed by the mean anomaly factor $\mathrm{f}_{\mathrm{M}}$, as introduced in McNaught and Asher (1999a).

The radial dispersion from the trail center occurs by the mechanism of non-isotropic scattering or thermal re-radiation of solar radiation, as discussed in Section 3.

It is assumed that the dispersion affects all three dimensions, one of which appears in $\mathrm{f}_{\mathrm{M}}$. The spread in the two other dimensions is assumed proportional to orbit number. This is because the rotation axis is expected to maintain its rough direction for several orbits giving cumulative spread effects. Further it is proportional to original $\Delta \mathrm{a}$ (difference of original semi major axis from that of the comet at ejection time). This comes from the fact that the non-regular radiation effects can be assumed to be proportional to the regular force. This is especially true for scattering, but less so for the seasonal Yarkovsky effect. The direct radial scatter is expected to be largely due to the scattering. 
The radial density $\rho_{\mathrm{r}}$ of the trails is assumed to be of the form:

$$
\rho_{\mathrm{r}} \sim\left(1+\Delta \mathrm{r}^{2}\right)^{-0.5 \mathrm{p}}
$$

where $\Delta \mathrm{r}$ is the difference in radial ecliptic plane crossing distances of Earth and meteoroid $r_{E}-r_{D}$, scaled according to:

$$
\Delta \mathrm{r}=\left(\mathrm{r}_{\mathrm{E}}-\mathrm{r}_{\mathrm{D}}\right) /(0.00059 \times \mathrm{n} \times \Delta \mathrm{a})
$$

where $\mathrm{n}$ is the number of orbits since ejection and $\left(\mathrm{r}_{\mathrm{E}}-\mathrm{r}_{\mathrm{D}}\right)$ and $\Delta \mathrm{a}$ are in astronomical units. Equation 6 was derived from the observed activity profiles of past meteor storms with the assumption, that the profile shape radially (from Sun) is the same as normal to the orbital plane. In equation 7 , the terms $n$ and $\Delta \mathrm{a}$ arise, because the trail width is expected to widen directly with $\mathrm{n}$ and also with an increase of $\Delta \mathrm{a}$, practically resulting in a widening with increasing distance from the comet. From published graphs of the 1966 storm (Jenniskens, 1995; Mason 1995; Brown 1999), the free parameter $p$ was derived as $p=2.7 \pm 0.7$. The scaling factor 0.0002 in Equation 7 was derived from the 1966 storm case, which had $\mathrm{n}=2$ and $\Delta \mathrm{a}=0.169$. In addition, the densities are assumed proportional to the distance from the center raised to the power -2.7 far away from the trailet center.

We now introduce the empirical function $f(\Delta a)$, which is the ZHR for a one-revolution central encounter having the original $\Delta \mathrm{a}$. Noticing further the mentioned spread (and dilution) directly in two dimensions with $\mathrm{n}$ and further with $\mathrm{f}_{\mathrm{M}}$, we get this expression for ZHR:

$$
\mathrm{ZHR}=\mathrm{fn}(\Delta \mathrm{a})\left(\mathrm{f}_{\mathrm{M}} / \mathrm{n}^{2}\right)\left(1+\Delta \mathrm{r}^{2}\right)^{-0.5 \mathrm{p}}
$$

In principle, there may be two ways to improve the $\Delta \mathrm{r}$ scaling Equation 7. One is to compare the dispersion scaling radial and normal to the orbital plane. The other is to get a mutual fit for $\mathrm{fn}(\Delta \mathrm{a})$ for central and non central passes (for example comparing storms in 1833 and 1966). A trial with the first would change the above from $\Delta \mathrm{r}=0.0002$ to $0.00025 \mathrm{AU}$. However, the parameter was left as originally assumed.

An approximation of $\mathrm{fn}(\Delta \mathrm{a})$ was derived from a fit of observed outbursts. The peak ZHR observations used are the same as used by McNaught and Asher (1999a). For one of the outbursts this was actually the only source available, while for others there was no clear reason to change those values. The course of this function with points derived from observations is shown in Figure 4.

The resulting predictions are given in Table I. The table lists the year of return, the number of revolutions since ejection (n), the difference in radial ecliptic plane crossing distances (the miss distance), the function $\mathrm{f}_{\mathrm{M}}$, the difference in semi-major axis, the predicted Zenith Hourly Rate, the predicted time of the peak (in solar longitude, J2000), the 
corresponding date, and some comments on what mechanisms may affect the characteristics of the shower.

\section{TABLE I}

\begin{tabular}{|c|c|c|c|c|c|c|c|c|c|}
\hline Year & Rev. & rE-rD & $\mathrm{fM}$ & delta a & Pred. ZHR & $\begin{array}{l}\text { Sol.long. } \\
2000.0\end{array}$ & $\begin{array}{l}\text { Date } \\
\text { Nov. }\end{array}$ & Time UT & Comments \\
\hline 2000 & 2 & -0.0012 & 0.55 & 0.30 & 215 & 235.270 & 17 & $07: 50$ & mostly faint \\
\hline 2000 & 4 & 0.00080 & 0.135 & 0.116 & 700 & 236.279 & 18 & $07: 50$ & \\
\hline 2000 & 8 & 0.00080 & 0.250 & 0.065 & 700 & 236.103 & 18 & $03: 40$ & $\begin{array}{l}\text { fM locally bigger, but } \\
\text { strongly affected by } A 2 \text { ef }\end{array}$ \\
\hline 2001 & 4 & 0.00025 & 0.135 & 0.144 & 6100 & 236.467 & 18 & $18: 22$ & \\
\hline 2001 & 5 & 0.00178 & 0.114 & 0.095 & 60 & 236.29 & 18 & $14: 10$ & \\
\hline 2001 & 6 & 0.00135 & 0.123 & 0.080 & 110 & 236.20 & 18 & $12: 00$ & \\
\hline 2001 & 7 & -0.00043 & 0.140 & 0.081 & 2000 & 236.115 & 18 & $09: 58$ & \\
\hline 2001 & 9 & 0.0001 & 0.260 & 0.043 & $1500-2000$ & 236.433 & \multicolumn{2}{|c|}{ rev:s $9,10,11$} & strongly affected by $\mathrm{A} 2$ ef \\
\hline 2001 & 10 & 0.0006 & 0.160 & 0.030 & 600 & 236.423 & \multicolumn{2}{|c|}{ ZHR comb. } & \\
\hline 2001 & 11 & 0.0004 & 0.160 & 0.026 & 260 & 236.425 & \multicolumn{3}{|c|}{ ab. 2500 to 3000} \\
\hline 2002 & 4 & -0.00004 & 0.148 & 0.174 & 7400 & 236.894 & 19 & $10: 44$ & \\
\hline 2002 & 5 & 0.00148 & 0.115 & 0.120 & 160 & 236.72 & 19 & $06: 45$ & \\
\hline 2002 & 7 & -0.00013 & 0.130 & 0.114 & 4500 & 236.612 & 19 & $04: 02$ & \\
\hline 2003 & $\begin{array}{l}15 / 14 \\
\mathrm{co} / \mathrm{me}\end{array}$ & $\begin{array}{l}-0.001 \text { to } \\
-0.003\end{array}$ & $\frac{1-2.5}{\text { extr.conf.! }}$ & 0.28 & 250 & $\begin{array}{c}230.69 \\
\text { to } 230.78+\end{array}$ & $\begin{array}{r}13 \\
! ! !\end{array}$ & 13 to 19 & $\begin{array}{l}\text { very special } \\
\text { also uncertain }\end{array}$ \\
\hline 2006 & 2 & -0.0002 & 0.470 & 0.94 & 50 & 236.618 & 19 & $04: 48$ & maybe strong teles- \\
\hline 2007 & 2 & -0.0004 & 0.560 & 1.06 & 30 & 236.107 & 19 & $22: 55$ & copic outbursts \\
\hline
\end{tabular}

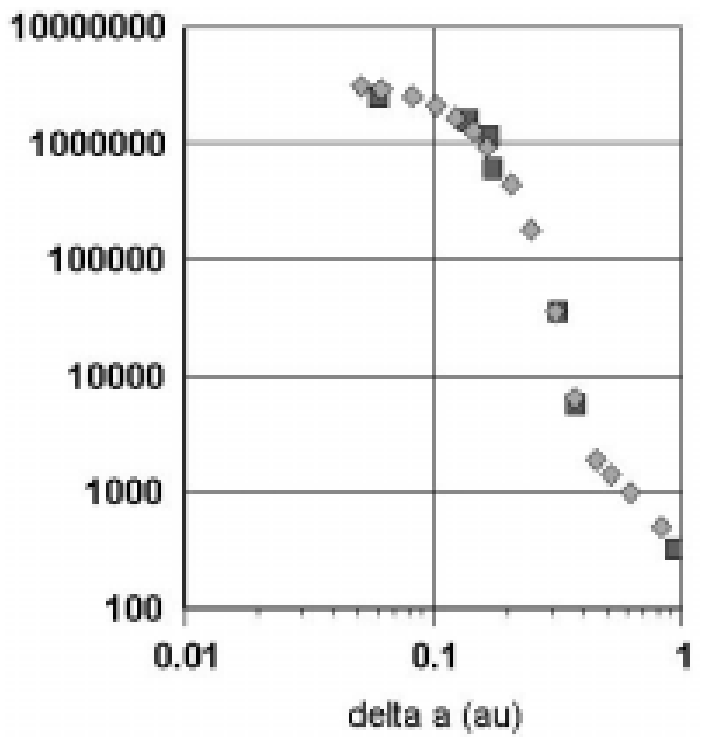

Figure 4. The graph shows the approximation of $\mathrm{fn}(\Delta \mathrm{a})$ from seven observed peak Zenith Hourly Rates. Squares are derived from observations of 1866, 1999, 1966, 1833, 1869, 1867, and 1969. Diamonds show the derived numerical approximation. 


\section{Discussion}

\subsection{THE A2 EFFECT}

For young trails, the A2 effect is expected to have a relatively small influence on the predictions. Even for not quite so old trails $(n \sim 4-6)$, this effect is probably dominated by planetary perturbations at large radial distances. For old trails, the A2 effect typically makes the ZHR smaller (by increasing the dispersion in the shower), but can also increase the rates by decreasing the miss distance $\left(\mathrm{r}_{\mathrm{E}}-\mathrm{r}_{\mathrm{D}}\right)$. The inclusion of this in the ZHR-formula was tested by assuming that it mostly affected the radial direction. However, there appear to be cases where it mostly shifts the longitude of the ecliptic crossing. Further, the effect is hard to scale, and frequently the situation seems to be very irregular, for example $\mathrm{f}_{\mathrm{M}}$ changing quite strongly with the applied effect. The $11 \mathrm{rev}$. 1903 outburst ZHR as compared to the prediction is very suggestive of the existence of this effect and in principle may be used for scaling. This was an encounter with small miss distance. The ZHR may have been a few hundred (Jenniskens, 1995). The model gives a postprediction value of around 3600 without assuming an A2 effect.

Even though this effect was not included in the ZHR formula, this has been to some degree considered in the predictions. For older trails ( 7 revolutions and up) this effect is in some cases assumed to reduce the ZHR, and this may increase the ZHR in the $n=10$ case in 2001. This effect also changes the timing, maybe up to hours for distant encounters. The effect on timing is not included in the table data, except in the special case in 2003, where this effect may help to bring particles near the Earth orbit a few hours after the nodal crossing. The suggested timing is stretched a few hours past the nodal crossing.

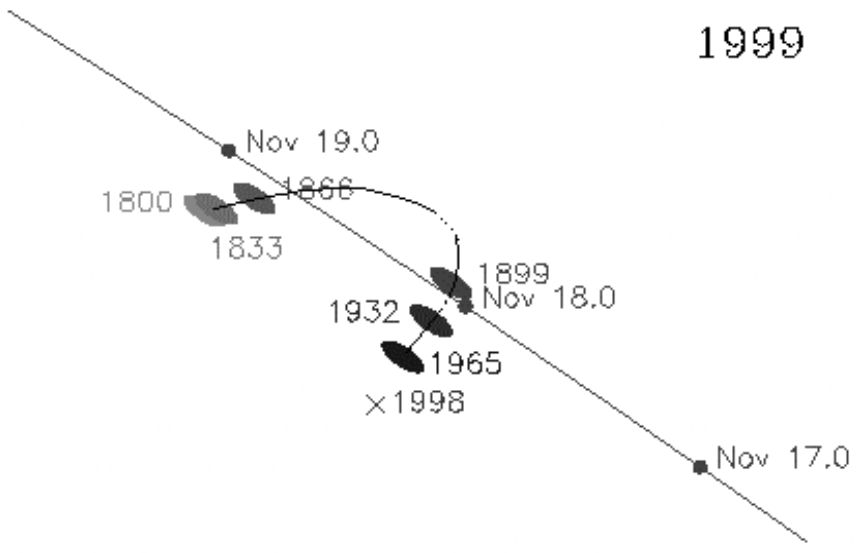

Figure 5. Drawing a smooth curve trough the trails in the order of $\mathrm{n}$ may be a practical means of timing far encounters such as with the 1866 trail. Original Figure by David Asher, reproduced and edited with permission. 


\subsection{POSSIBLE PRACTICAL MEANS OF TIMING DISTANT ENCOUNTER OUTBURSTS}

While looking at the David Asher ecliptic plot for the year 1999, we recognized a special behavior in the timing. Even though the region around 16 to 20 UT Nov 18 is not very well mapped by observations, it appears as if the distant encounter with the $5 \mathrm{rev}$ trail would have happened some hours prior to the nodal encounter (Jenniskens et al., 2000). A treatment as in Figure 5 may explain the timing. It is as if there were some kind of sheets existing between the trails. This is probably explained by A2 type effects. This may suggest that some particles are more affected than would otherwise be anticipated.

\subsection{CONSIDERATIONS ON THE WIDTH (DURATION) OF AN OUTBURST}

In principle, this model should also give the width of outbursts at least for close encounters. Some first exploration of parameter space was made, but not a thorough treatment to present here. For young trails, spreading in length almost uniformly with orbit number, the width should be about proportional to the time interval from the passage of the parent comet. This relation seems not to hold for the observed 1969 outburst with large original $\Delta \mathrm{a}$.

The comparison of observations with the model is greatly complicated by the effect of the miss distance. Before the 1999 storm, we assumed that the profile of the trails in all directions of cross section is the same. This would lead to a purely mathematical means of deriving the change of the width according to the miss distance. The observed duration of the 1999 storm shows that this assumption led to an overly large duration prediction. The conclusion was that the assumptions on the cross section are not true. Actually, the observed behavior may give a further clue to the cause of the dispersion. Further modeling of these radiation pressure phenomena is suggested, by considering, simultaneously, the dispersion near the descending node radially and normal to plane. With this modeling, the observed relative widths radially and normal to orbital plane should also be in agreement. A comparison of observed duration (further changed to a normal-tothe-orbit-plane value) with the radial dependency according to the model, gives the radial spread to be about 2.5 times the normal for young trails. Because of the inclination of the cometary orbit, the scatter happens to be roughly equal in the ecliptic plane both radially and along the direction of Earth's motion.

Jenniskens et al. (2000) deal with the profile shape and width of the 1999 Leonid storm as measured very precisely from airborne observations during the Leonid Multi-Instrument Aircraft Campaign. They find a good fit with a Lorentz curve shape. Interestingly, the distribution in Equation 6 does also have a Lorentz shape when $p=2$ (we have it $2.7 \pm 0.7$ ). This paper's ZHR model could be easily transformed to use this Lorentz form. Of course, the $\mathrm{fn}(\Delta \mathrm{a})$ would need to be determined accordingly. The effect on the predicted ZHRs would 
probably not be too much if the observations are concentrated in the same $\Delta \mathrm{r}$ range as the predicted cases.

In addition, Jenniskens et al. (2000) find that the whole trailet pattern is shifted by about $0.0003 \mathrm{AU}$ from the assumed dependency and plot of observed meteor storm profile widths. This conclusion depends on the assumption that the Lorentz shape applies to the radial distribution as it does to the normal distribution. As pointed out by Jenniskens et $a l$., this can not be proven yet due to lack of data. For the widening not to happen as the simple assumption in Jenniskens et al., requires a more rapid decline at angles between radial and normal from the trails. Interestingly, our model finds that the profile can have this Lorentz shape in both radial and normal directions to the plane, and yet not widen as expected. For example, if y represents the radial distance from trail center and $x$ that normal to the orbital plane, then these are already scaled according to the mutual central half-widths (to get a simplified expression). If the ZHR is of the type:

$$
\mathrm{ZHR}(\mathrm{x}, \mathrm{y})=\mathrm{ZHR}_{\max } /\left(1+\mathrm{x}^{2}+\mathrm{y}^{2}\right)
$$

then the observed Lorentzian broadening in the $x$-direction (with increasing pass distance y) does arise. Here, $\mathrm{ZHR}_{\max }$ is the maximum corresponding to the center of the trail, and not the observed maximum. However, the same broadening will not arise when the dust density in the trailet has the form:

$$
\begin{aligned}
\mathrm{ZHR}(\mathrm{x}, \mathrm{y}) & =\mathrm{ZHR}_{\max } /\left(\left(1+\mathrm{x}^{2}\right)\left(1+\mathrm{y}^{2}\right)\right) \\
& =\mathrm{ZHR}_{\max } /\left(1+\mathrm{x}^{2}+\mathrm{y}^{2}+\mathrm{x}^{2} \mathrm{y}^{2}\right)
\end{aligned}
$$

although the distribution is of Lorentz form both in the $\mathrm{x}$ and $\mathrm{y}$ directions, but not at intermediate cross sections. The last form shows that with this, the decline is more rapid into other than axial directions. In our model (Equation 8), the expressions in parentheses, either $\left(1+\mathrm{x}^{2}\right.$ $\left.+\mathrm{y}^{2}\right)$ or both $\left(1+\mathrm{x}^{2}\right)$ and $\left(1+\mathrm{y}^{2}\right)$, should be raised to the power $\mathrm{p} / 2=$ $2.7 \pm 0.7 / 2=1.35 \pm 0.35$.

If there is scatter in q caused "somewhere", and the scatter normal to the plane is caused somewhere else, and these are sufficiently independent from each other, then there doesn't arise the otherwise expected dependency of outburst duration with miss distance. The observations are suggestive that there is no or little dependency of duration on the miss distance to somewhat out of the trail centers, but there is dependency at larger miss distances.

It is expected that one key factor with the dependency of the width is that the ecliptic crossing happens near perihelion. So angular momentum changes normal to the plane near aphelion, where the efficiency of non-isotropic scattering of solar radiation is high, do not efficiently cause scatter normal to the orbital plane (near perihelion), whereas in-plane effects there do affect the change of q. The seasonal Yarkovsky effects at a distance of roughly 2 AU may be mainly responsible for the scatter normal to the plane. Both these depend on 
the direction of the spin axis. However, it is not known without special modeling if these effects are independent enough to explain the observations.

\subsection{UPCOMMING ENCOUNTERS}

Let us examine the predictions in Table I. As noticed before (Kondrat'eva and Reznikov, 1985; McNaught and Asher, 1999; Lyytinen, 1999), very strong meteor storm encounters are still to come. Especially in 2001 and 2002 rates can go up to ZHR $=6,000$ or almost twice the level of activity of the 1999 meteor storm. That event has now $\mathrm{ZHR}=3,200$ in our model, not too different from the observed value of $\mathrm{ZHR}=3,700$ (Arlt et al., 1999). The ZHR value of 2,500 to 3,000 in the line 2001 and 11 rev. means the combined value of trails with 9, 10 and 11 revolutions.

The first encounter ahead is that of the year 2000. In both the $n=4$ and 8 rev. cases, the trail centers will pass inside the Earth's orbit. It has been the other way (outside Earth's orbit) with all the observed outbursts, and we caution that this supposed symmetry (as in the model) in $r_{E}-r_{D}$ may not be true. Also, the 2000 encounter with the $n=8$ rev. trail is actually with a "piece" of trailet cut on both sides by perturbations of earlier passes near the Earth. By purely gravitational solution, the mean anomaly factor seems to be locally quite high, but it is expected that the A2 effect smoothens the trail fragment into a longer piece in space. In that case, the A2 effect does not seem to help (applied in either way) bring the particles closer to the Earth. In the prediction table, the mean anomaly factor has been dropped into about one third of the purely gravitational local value, but this is not much more than guessing (except that it does need reduction).

Further ahead, we expect that the encounters with young trails, mainly in 2006 and 2007, will be vastly more abundant in smaller particles (about one fourth of a $\mathrm{mm}$ in diameter), which will lower the rates in less perfect conditions. The special case in 2003 may also belong to this group.

The closest pass of the one-rev trail from 1965 (not included in the prediction table) happens in 2005 at about $0.0006 \mathrm{AU}$, whereby the trailet is passed inside the Earth's orbit. This may give practically no visual meteors, but may give a brief outburst of $0.1 \mathrm{~mm}$ particles instead that are a target of telescopic observations. The miss distance from the trail center is only about 2.5 times the geosynchronous distance. The geocentric nodal crossing is at solar longitude 235.569. This is on Nov 17 at about 21:52 UT (2005). The encounter with geosynchronous satellites on the day side will be up to about a half-hour later, depending on the exact location of the satellite.

Looking back in time, the 1998 "storm component" demonstrates a large dispersion of dust perpendicular to Earth's orbit. Considering the miss distances for various trailets, it would seem that the "storm component" was most likely caused by the three revolution trail (1899). This trail would have passed at about 0.004 AU from the Earth's orbit. 
On the other hand, this trail had a gap (caused by perturbations of a near pass by Earth in 1965) of about one month in just the position of the encounter in 1998. This region of the trail should have been empty. The trail from 1932 would have passed at $0.005 \mathrm{AU}$, and that from 1965 at almost at 0.007 AU. Because of the near pass of Jupiter around the 1899 return, the nodes of earlier trails don't fit at all. On the other hand, the presence of particles in this region can be explained if there exists quite a large A2 effect, a non-gravitational period change.

\section{Summary}

Considering the very good post-predictions of historic Leonids storms and the successful prediction of the 1999 storm, with the trail models as in Kondrat'eva and Reznikov (1985), McNaught and Asher (1999), and Lyytinen (1999), it is reasonable to expect that good timing predictions for future outbursts can now be made. The strength of outbursts is more difficult to predict. In this paper, we addressed various aspects that can affect the dust density (as reflected in the Zenith Hourly Rate). A semi-empirical model was built for the dust density in the trails that gives a good fit to past observations. It is therefore expected that predictions for future meteor storm encounters will also not be too far off. The main uncertainty is the assumed symmetry in the radial dispersion to both sides of the trails. Also, there are no observations yet of encounters with old trails from five to ten revolutions ago. The radiation pressure effects are found to be strong enough to explain the total observed dispersion away from the trail centers. A better understanding of these effects needs further attention.

\section{Acknowledgments}

We thank David Asher for support of the research by enabling direct comparisons of integrations, and discussions on the treatment of radiation pressure effects. This work was partially sponsored by Meta Research. We thank the editor for numerous improvements of the manuscript. This work furthers the goals of the Pro-Amat Working Group of IAU C22. Editorial handling: Peter Jenniskens.

\section{References}

Arlt, R., Bellot Rubio, L., Brown, P., and Gyssens, M.: 1999, WGN, Journal of the IMO 27, 286-295.

Brown, P.: 1999, Icarus 138, 287-308.

Jenniskens, P.: 1995, Astron. Astrophys. 295, 206-235.

Jenniskens, P., Crawford, C., Butow, S., Nugent, D., Koop, M., Holman, D., Houston, K., Kronk, G., and Beatty, K.: 2000. Earth, Moon and Planets, 82-83, 191-208.

Kondrat'eva, E. D. and Reznikov, E. A.: 1985, Sol. Syst. Res. 19, 96-101. 
Kondrat'eva, E. D., Murav'eve, I. N., and Reznikov, E. D.: 1997, Sol. Syst. Res. 31, 489-492.

Kresak, L.: 1976, Bull. Astron. Inst. of Czechoslovakia 27, 35-46.

Lyytinen, E.: 1999, Meta Research Bulletin 8, 33-40.

Mason, J.W.: 1995, Journal of The British Astronomical association 105, 219-235.

McNaught, R.H. and Asher, D.J.: 1999a, WGN, Journal of the IMO 27, 85-102.

McNaught, R.H. and Asher, D.J.: 1999b, Meteoritics Planet. Sci. 34, 975-978.

Nakano, S.: 1998, Minor Planet Circular 31070

Olsson-Steel, D.: 1987, MNRAS 226, 1-17.

Pavel, M.: 1955, J. Atmos. Terr. Phys. 2, 168-177.

Rubincam, D. P.: 1995, J. Geophys. Res. 100, E1, 1585-1594.

Van Flandern, T.: 1981, Icarus 47, 480-486.

Van Flandern, T.: 1999, Dark Matter Missing Planets \& New Comets, North Atlantic Books, Berkeley, CA, $2^{\text {nd }}$ edition, chapter 10.

Vokrouhlicky, D. and Broz, M.: 1999, Astron. Astrophys. 350, 1079-1084.

Yeomans, D.K., Yau, K.K., and Weissman, P.R.: 1996: Icarus 124, 407-413. 